\title{
AUTOMATIC CONSTRUCTION SITE MANAGEMENT WITH AERIAL AND CLOSE RANGE PHOTOGRAMMETRY
}

\author{
Liao, Ching-Lung \\ Director General, Bureau of Taiwan High Speed Rail, Ministry of \\ Transportation and Communications
}

\begin{abstract}
Aerial photos and engineering blueprints developed in a related database can be used for facilitating construction site management by their distinct contrast features. The contrast would be much more distinguishing through the 3D spatial factors. The 3D spatial factors are mostly from the aerial photos and close range image by means of image processing and feature extraction. Geography Information System (GIS) deployed on ArcView serves as an interfacing port between user and database for information inquiry and analysis. All the spatial data are constructed into as multi-layered vector structure. Main tasks of the interface are the extraction of multi-dimension temporal and spatial features, and their corresponding factors that can be used to verify the actual construction progress on site.
\end{abstract}

\section{Introduction}

The highly advanced technique of computer and information system has made the automatic process in many aspects possible. Fast CPU, huge storage hard disk, compact disks, and more than Giga Bytes RAM have made the high-resolution image processing feasible on regular personal computer. Some applications of aerial photos and ground pictures to the automatic construction site monitoring system are introduced in this paper. The key issue is to gather the temporal and spatial change of certain area with aerial photos or ground pictures. After appropriate processing, each pixel on those images possesses a unique 3 dimensional coordinates which are acquired by means of aerial triangulation calculation in the ground base Global Positioning System.

Once ground coordinates of certain figure are obtained, the associated properties can be linked. The GIS is used to handle both temporal and spatial data with precise ground coordinates. To utilize such a system for construction site management it needs to compare the 'actual image taken at later stage' with the original image taken previously. By comparing the difference between these two images, the change within such period of time can be gathered. These images can also be used for verifying the actual construction progress because they are at the same coordinates, but taken in the different time periods.

\section{Handling Processes}

For every photogrammtery work, several precise ground control points are required. Those control points can be obtained marked by the special marker on chosen locations before the photos are taken or reoccupied several distinct points for accurate coordinates after photos are taken. Main work is to find the true geometry of each aerial photo that constrained by the interior geometry (pixels with photo image) and compared to the exterior geometry (ground control points coordinates)(wolf and brinker, 1994). Once the orthofication of each image has been complied, the exact coordinates of each pixel on images and the coordinates of certain target region or buildings can be gathered. The working flow is shown in figure 1. 

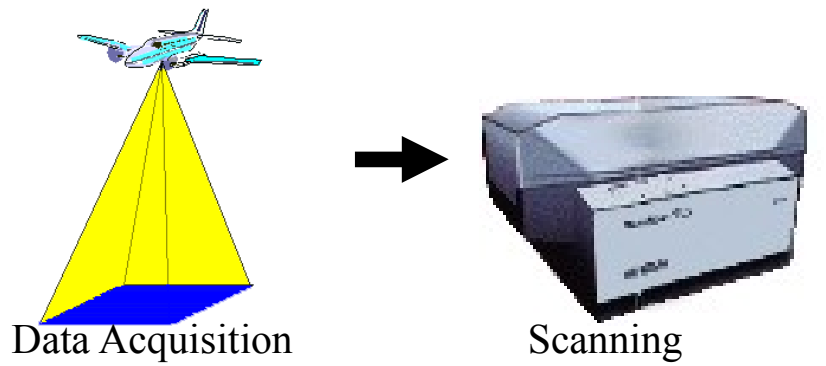

Scanning

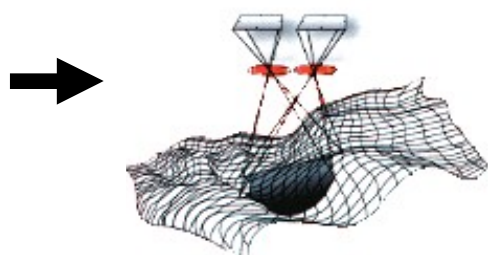

Measurement and Triangulation

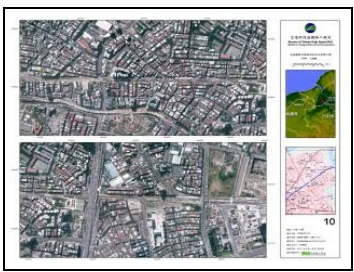

Output

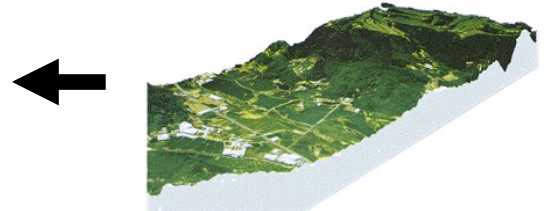

Orthophoto Generation

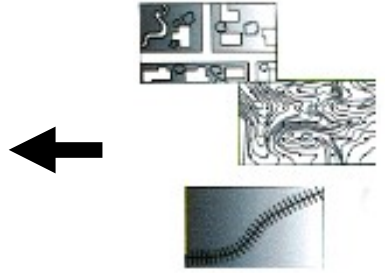

Data Collection

Figure 1: The flow chart of orthophoto processing, (source from Intergraph SSK workstation reference guide).
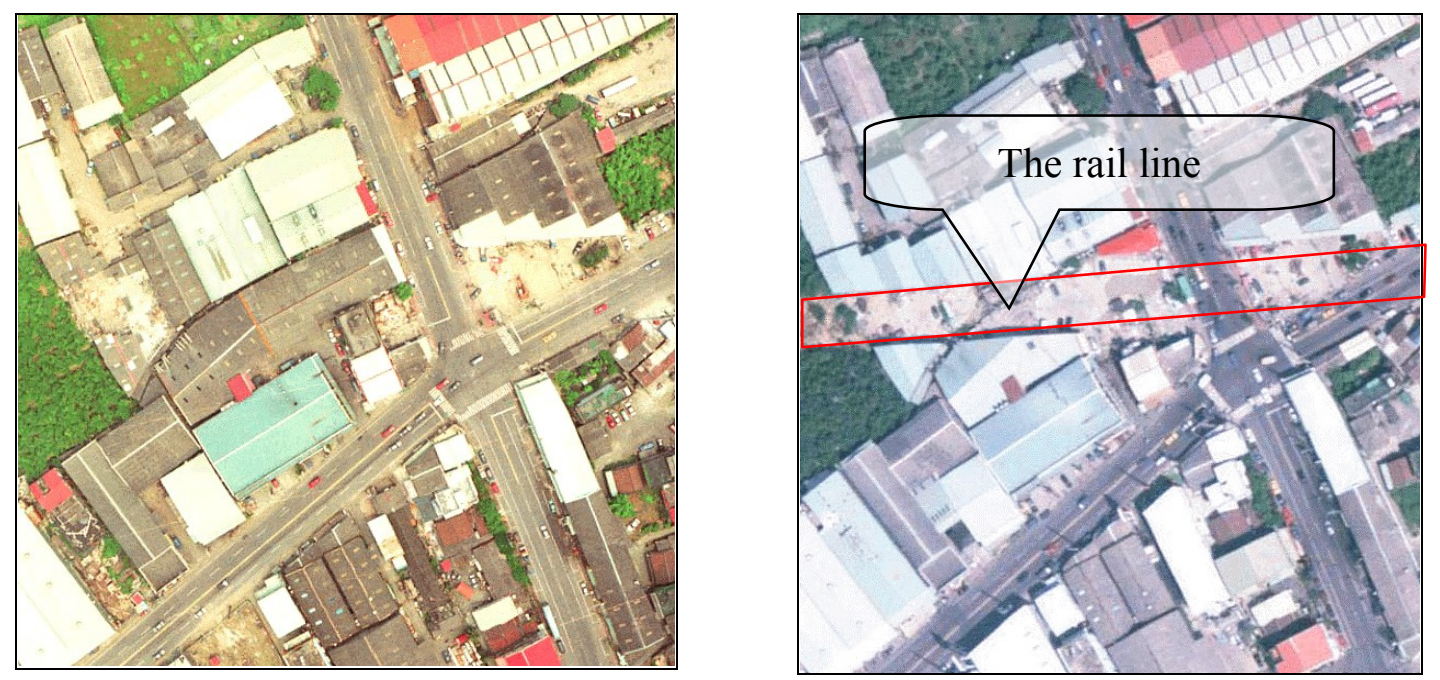

Figure 2 .

Ground

condition before (left) or after (right) demolishing $17 \mathrm{~K}+300$.

Applications

Figure 2 shows the images of ground condition in certain area on the Taiwan High Speed Rail line before and after the clearing work for the right of way.

From the figure 2, it is clear to see the in-situ conditions at these two stages. Before the clearing work the site condition is quite normal; after the clearing work the ground surface is altered due to the demolition and rebuilding to provide the required right of way. By comparing these two images in large scale, we can clearly verify and conclude the completion of the demolishing and rebuilding work. This is an excellent example to demonstrate the power of image in real time and true situation verification.

Another example of the application is to have general information for target region before the planning or the construction stage. The contractor can use aerial photo as shown in Figure 3 to evaluate possible alternative access roads to a construction site with complicate photography or environment such as the SICHI depot of the Taiwan High Speed Rail project. The progress of construction can be seen at a certain stage from this image. 


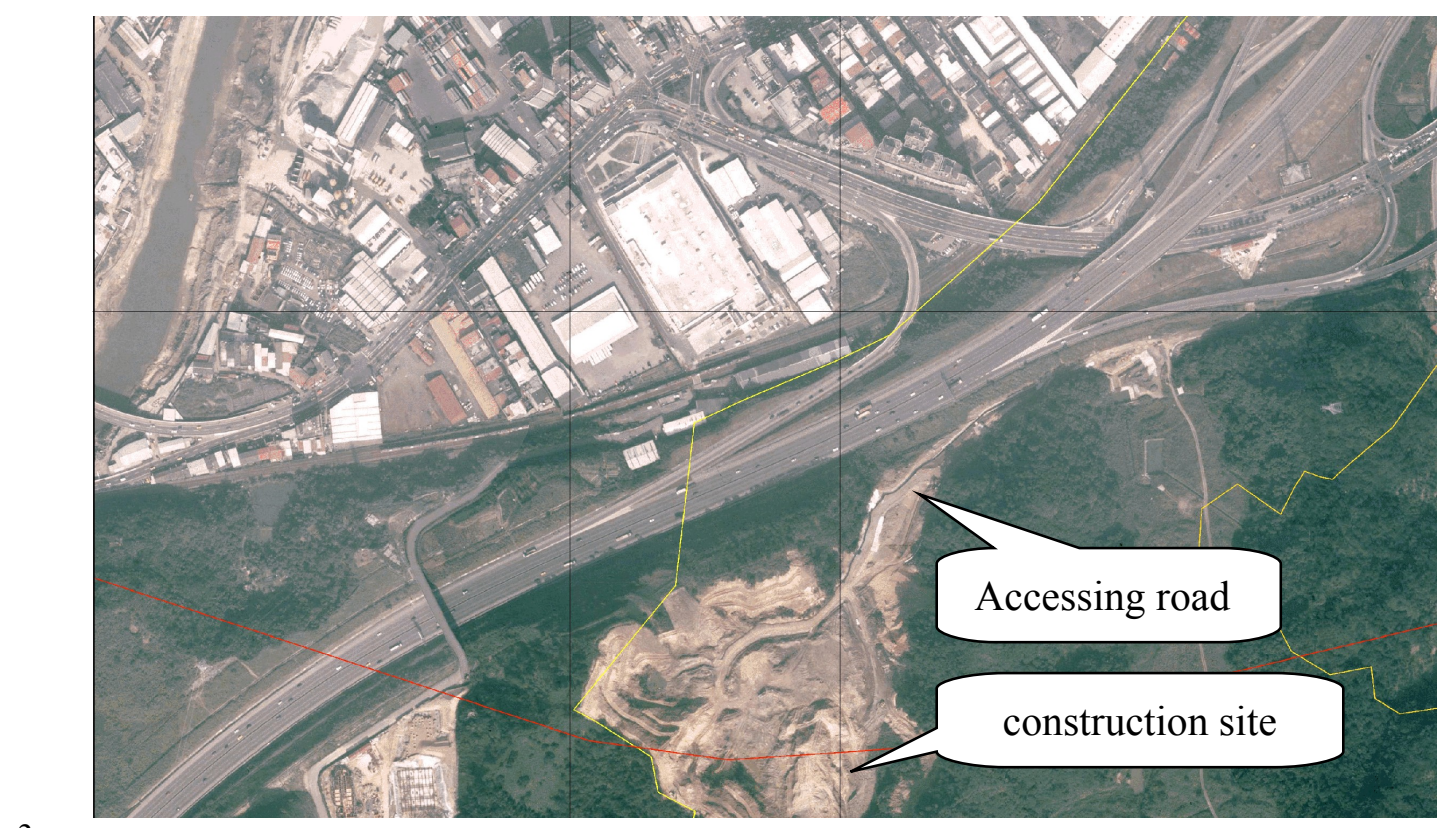

Figure

3:

of construction site and possible accessing roads.
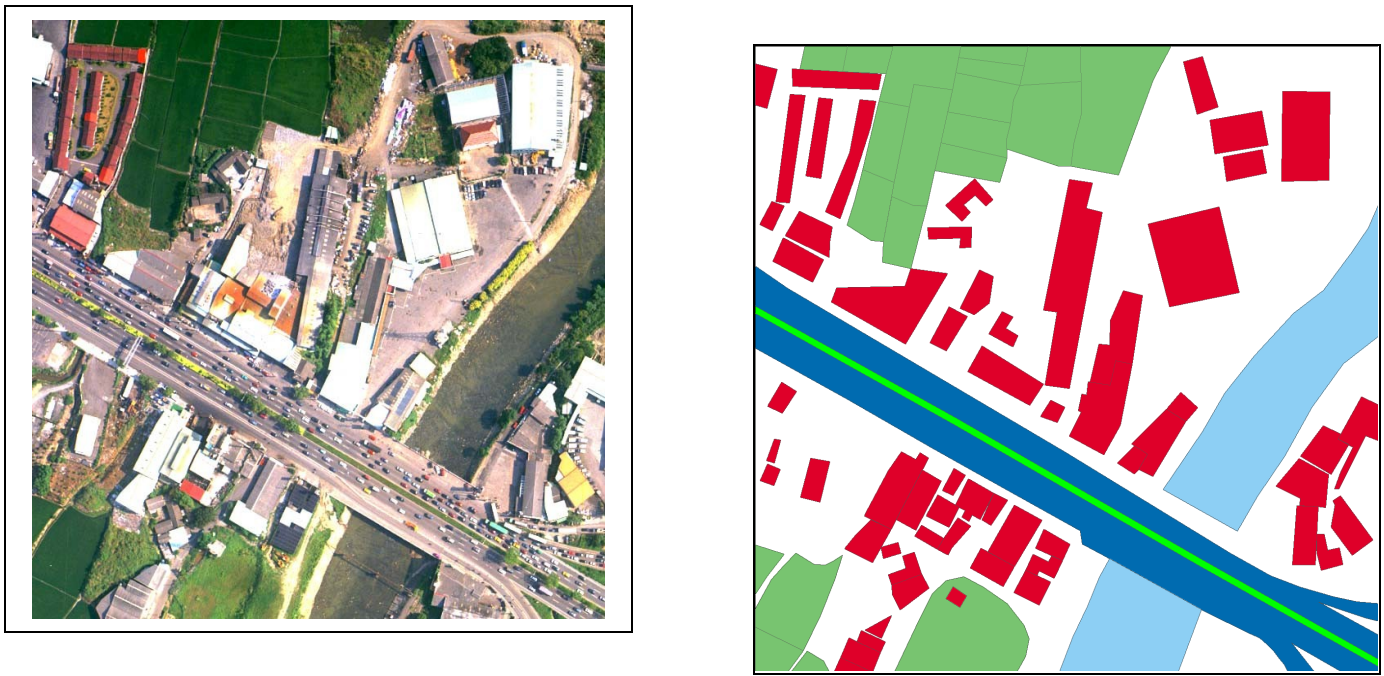

Figure 4: The extracted features on top of aerial photos.

Once the orthophoto is produced for certain regions, it is straight -forward to extract the distinct features directly from images, such as road, river, property boundaries and so on. Figure 4 shows an example.

The vector data of such features can be transported into Geography Information System (GIS) that serves as spatial information base. With detailed property information updated at various stages of time, user can snatch the temporal variation of particular region such as the land usage, new development and environmental changes. Of course, user can always input the construction plan for particular project into these 3S (GPS, GIS, RS) systems and use this technique for data gathering and condition simulation. RS means Remote Sensing.

The close range photogrammtery is another useful technique for the automatic construction site management. It takes photos on the ground instead of from the sky through airplanes. Figure 5 is a typical example of this technique. Two different images taken on the ground with different angles have linked together to set up the complete 3D model for a 
chemical processing plant.

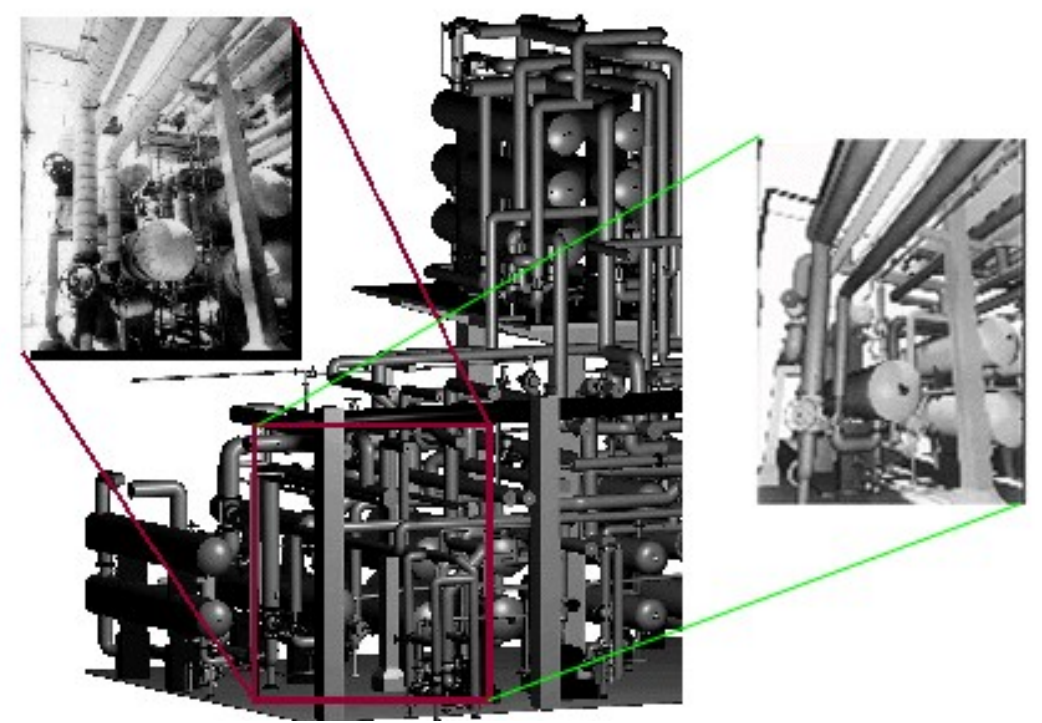

Figure 5: 3D model constructed by two close range images, (source from www.vexcel.com/fotog/demo/fotog.into.PPT).

\section{Conclusions}

By the aid today's advanced PC technology, user can easily accomplish many tasks that are almost impossible 5 years ago. One of the key examples is the progress of aerial and close range photogrammtery. With the deployment of IKONOS satellites, near real time and high resolution image is accessible for almost everyone that is willing to pay. If the data/information acquiring platform such as airship, remote control airplane can be launched in Taiwan, then real time information can be gathered within required resolution and precision. Once the data gathering has been erected, it is just a matter of time to obtain the information for the user or computer. Consequently, the use of photogrammtery images will be an effective and efficient way to monitor the progress of certain construction site, or to evaluate condition of remote area.

\section{References}

1. Intergraph SSK workstation reference guide lchapter $1 \backslash$ general introduction.

2. Wolf, Paul; R. C. Brinker, Elementary Surveying, $9^{\text {th }}$ edition, HarperCollins College Publishers, 1994, pp: 760.

3. www.vexcel.com/fotog/demo/fotog.into.PPT, web site. 